\title{
View outside the box: Supramolecular synthon and intermolecular interactions as a directing tool for shaping magnetic behaviour in design of supramolecular architectures of copper(II) complexes
}

\author{
N. Penić ${ }^{1,2}$, D. Pajić ${ }^{2}$ M. Đaković ${ }^{1}$ \\ ${ }^{I}$ Department of Chemistry, Faculty of Science, University of Zagreb, \\ ${ }^{2}$ Department of Physics, Faculty of Science, University of Zagreb \\ penic@phy.hr
}

In crystal engineering hydrogen and halogen bonds have proven to be very valuable crystal engineering tool for design of supramolecular architectures by self-assemblies of small building blocks, shaping their final architectures and determine the resultant topology and ultimately controlling many physical properties. [1] A number of supramolecular synthetic strategies to harness their potential have already been developed, but only for purely organic system. Although metal-organic supramolecular assemblies exhibit many technologically important properties, their design is often difficult to predict because introduction of metal cations and chargebalancing entities into metal-free solids commonly disrupt well-established connectivity of the key functional groups.[2] This is especially pronounced for magnetic metal-organic systems where magnetic behaviour not only depends on fine tuned parameters in the crystal packing but as well on the functional group, nature of the acceptor (A) and donor (D) atoms, lengths and angles of noncovalent interactions. When all of this is taken into account, targeting supramolecular architectures with desired magnetic properties becomes even more difficult and multiplex. Therefore, in those systems hydrogen and halogen bonds are rarely explored as magnetic exchange pathways or as a crystal engineering tool for directing magnetic behaviour. As well as molecular interactions, in field of molecular magnetism, metal-organic systems are not even approximately investigated as a miscellaneous copper oxide compounds, especially compounds with pyrazine and pyridine based ligands in which copper is bridged by halogen element. So far it is known that pyrazine and pyrazine derivates can be mediators of magnetic exchange within dimers, linear chains and two-dimensional lattices, and they are used in preparation of low-dimensional magnetic materials.[3] However, some insight in functional group effects on magnetic exchange of these systems in literature is not observed.

In order to understand the magnetic behaviour of crystalline coordination compounds with general formula $(n-R p z / p y m / p y) C u X_{2}$ and correlate structural features (in particular, functional groups, chemical linkages, bond length and angles) to magnetic exchange, we presented statistical and magneto-structural analysis of crystallography database and prepared a series of 1D polymeric chain copper(II) halides with pyrazine-, pyrimidine- and pyridine based ligands bearing the lactam or halogen functionality as a supramolecular synthetic vector. For all obtained coordination compounds $\left(\left[\mathrm{CuCl}_{2}\left(2-\mathrm{NH}_{2} \mathrm{pz}\right)_{2}\right]_{n},\left[\mathrm{CuCl}_{2}(2-\mathrm{pyz})_{2}\right]_{n},\left[\mathrm{CuCl}_{2}(4-\mathrm{pym})_{2}\right]_{n}\right.$, $\left[\mathrm{CuBr}_{2}(4-\mathrm{pym})_{2}\right]_{n},\left[\mathrm{CuBr}_{2}(3-\mathrm{Clpy})_{2}\right]_{n},\left[\mathrm{CuBr}_{2}(3-\mathrm{Brpy})_{2}\right]_{n}$ and $\left.\left[\mathrm{CuBr}_{2}(3-\mathrm{Ipy})_{2}\right]_{n}\right)$ temperature dependence of magnetization $\mathrm{M}(\mathrm{T})$ was measured using SQUID magnetometer in the temperature range 2-300 K. Linear dependence between magnetization and magnetic field allows usage of the linear magnetic susceptibility, $\chi$. In accordance with crystal structure, we applied approach of BonnerFischer and modelled entire $\mathrm{M}(\mathrm{T})$ curves for all obtained compounds using spin chain of antiferromagnetically interacting neighbouring $\mathrm{Cu}^{2+}$ ions along structural chains. [4] These results are compared and discussed within structural features influence on magnetic superexchange $\mathrm{J}$.

[1] Bernstein J.; Crystal growth, polymorphism and structure-property relationships in organic crystals properties, J. Phys. D: Appl. Phys. 1993, 26, B66

[2] Desiraju, G.R. Crystal engineering: a holistic view, Angew. Chem. Int. Ed. 2007, 46, 8342-8356

[3] Herringer S. N.; Longendyke A. J.; Turnbull M. M.; Landee C. P.; Wikaira J. L.; Jameson G. B.; Telfer S. G. Synthesis, structure, and magnetic properties of bis(monosubstituted-pyrazine)dihalocopper(II) Dalton Trans. 2010, 39, 2785-2797

[4] O. Kahn, Molecular magnetism, Wiley-VCH, 1992.

Keywords: supramolecular assemblies of copper(II) complexes, antifferomagnetic spin chains, intermolecular interactions 